\title{
Third Year of Survey Data Shows Continuing Benefits of Medicaid Expansions for Low-Income Childless Adults in the U.S.
}

\author{
John Cawley, Ph.D. ${ }^{1,2,3}$, Aparna Soni, M.A. ${ }^{4}$, and Kosali Simon, Ph.D., \\ 'Department of Policy Analysis and Management, Cornell University, Ithaca, NY, USA; '2Department of Economics, Cornell University, Ithaca, NY, \\ USA; ${ }^{3}$ National Bureau of Economic Research, Cambridge, MA, USA; ${ }^{4}$ Department of Business Economics and Public Policy, Indiana University - \\ Bloomington, Bloomington, IN, USA; ${ }^{5}$ School of Public and Environmental Affairs, Indiana University - Bloomington, Bloomington, IN, USA.
}

\begin{abstract}
BACKGROUND: The Affordable Care Act (ACA) of 2010 incentivized states to expand eligibility for their Medicaid programs. Many did so in 2014, and there has been great interest in understanding the effects of these expansions on access to health care, health care utilization, and population health.
\end{abstract}

OBJECTIVE: To estimate the longer-term (three-year) impact of Medicaid expansions on insurance coverage, access to care, preventive care, self-assessed health, and risky health behaviors.

DESIGN: A difference-in-differences model, exploiting variation across states and over time in Medicaid expansion, was estimated using data from the Behavioral Risk Factor Surveillance System (BRFSS) for 2010-2016.

PARTICIPANTS: Low-income childless adults aged 1964 years in the BRFSS.

MAIN MEASURES: Outcomes included insurance coverage, access to care, several forms of preventive care (e.g., routine checkups, flu shots, HIV tests, dental visits, and cancer screening), risky health behaviors (e.g., smoking, alcohol abuse, obesity), and self-assessed health.

KEY RESULTS: The previously documented benefits of Medicaid expansions on insurance coverage, access to care, preventive care, and self-assessed health have persisted 3 years after expansion. There was no detectable effect on risky health behaviors.

CONCLUSIONS: The Affordable Care Act was motivated in part by a desire to increase health insurance coverage, improve access to care, and increase use of preventive care. The Medicaid expansions facilitated by the ACA are helping to achieve those objectives, and the benefits have persisted 3 years after expansion.

KEY WORDS: Affordable Care Act; Medicaid; health insurance; preventive care; health behaviors.

J Gen Intern Med 33(9):1495-7

DOI: $10.1007 / \mathrm{s} 11606-018-4537-0$

(c) Society of General Internal Medicine 2018

\section{INTRODUCTION}

The effects of the 2014 Medicaid expansions facilitated by the Affordable Care Act are the subject of numerous studies that

Received February 5, 2018

Revised March 27, 2018

Accepted June 8, 2018

Published online June 25, 2018 examine the first 2 years of data (2014-2015) since the expansion. ${ }^{1-6}$ These studies are informative about the early effects of the expansions, but it may have taken time for changes in enrollment, utilization, and health behaviors to occur. This paper presents the first results based on 3 years of postexpansion data (2014-2016) that measure the nationwide impact of Medicaid expansion on insurance status, access to care, preventive care, risky health behaviors, and self-assessed health.

\section{METHODS}

We estimated difference-in-differences (DD) models in which treatment was the state expansion of eligibility for its Medicaid program. The dependent variables concerned insurance status, access to care, preventive care, risky health behaviors, and self-assessed health. ${ }^{2}$ The models controlled for individual demographic characteristics (including age, sex, educational attainment, race/ethnicity, marital status, employment status, household size, cell phone sample), state unemployment rate, state of residence, and year of interview.

The DD models were estimated using data from the Behavioral Risk Factor Surveillance System (BRFSS) for 20102016; the sample is representative of national demographics. We restricted the sample to those most targeted by the expansions: low-income childless adults aged 19-64. The sample size was $N=80,200$, although the sample size differed across outcomes due to missing data. The model utilized BRFSS survey weights, and robust standard errors were clustered at the state level. Two-sided hypothesis tests were conducted, with statistical significance defined at the 5\% level. All analyses were conducted in Stata 14. This study was deemed exempt from review by Indiana University's institutional review board.

\section{RESULTS}

The Table 1 lists the coefficient estimates from the DD models, i.e., the estimated impact of Medicaid expansion on the outcome. Dividing the coefficients (in column 1) by the mean of the outcome prior to expansion (in column 3) provides the percent change in the outcome. Over the 3 years since 
Table 1 Difference-in-Differences Estimates of the Impact of Medicaid Expansion, 2010-2016.

\begin{tabular}{|c|c|c|c|}
\hline & DD estimate (1) & $P$ value (2) & Pre-expansion mean (3) \\
\hline \multicolumn{4}{|l|}{ Panel 1: Insurance } \\
\hline Have insurance & $0.103[0.067,0.140]$ & $<0.001$ & 0.617 \\
\hline \multicolumn{4}{|l|}{ Panel 2: Access to care } \\
\hline Have personal doctor & $0.025[0.001,0.049]$ & 0.044 & 0.636 \\
\hline Cost a barrier to care & $-0.036[-0.058,-0.014]$ & 0.002 & 0.338 \\
\hline \multicolumn{4}{|l|}{ Panel 3: Preventive care } \\
\hline Routine checkup & $0.024[-0.008,0.056]$ & 0.137 & 0.580 \\
\hline Flu shot & $0.008[-0.017,0.034]$ & 0.517 & 0.282 \\
\hline HIV test & $0.025[0.0003,0.050]$ & 0.048 & 0.425 \\
\hline Dentist visit & $0.025[0.0001,0.050]$ & 0.050 & 0.457 \\
\hline Clinical breast exam & $-0.009[-0.061,0.044]$ & 0.738 & 0.570 \\
\hline Pap test & $-0.011[-0.066,0.043]$ & 0.677 & 0.470 \\
\hline Mammogram & $0.012[-0.034,0.058]$ & 0.601 & 0.549 \\
\hline \multicolumn{4}{|l|}{ Panel 4: Health behaviors } \\
\hline Current smoker & $-0.009[-0.027,0.008]$ & 0.281 & 0.330 \\
\hline Heavy drinking & $-0.007[-0.017,0.003]$ & 0.151 & 0.064 \\
\hline Binge drinking & $0.005[-0.018,0.029]$ & 0.654 & 0.178 \\
\hline Exercise & $0.028[-0.007,0.062]$ & 0.112 & 0.661 \\
\hline BMI $(\times 100)$ & $21.604[-15.197,58.405]$ & 0.244 & 2816.1 \\
\hline Obese & $0.012[-0.005,0.030]$ & 0.158 & 0.319 \\
\hline \multicolumn{4}{|l|}{ Panel 5: Self-assessed health } \\
\hline General health $\left[{ }^{1-5}\right]$ & $0.089[0.050,0.129]$ & $<0.001$ & 2.923 \\
\hline Number days in past month mental health not good & $-0.704[-1.196,-0.211]$ & 0.006 & 8.143 \\
\hline Number days in past month physical health not good & $-0.475[-0.980,0.030]$ & 0.065 & 8.130 \\
\hline Number days in past month poor health prevented usual activities & $-0.969[-1.563,-0.375]$ & 0.002 & 9.629 \\
\hline
\end{tabular}

Authors' calculations based on BRFSS data from 2010 to 2016. The sample is restricted to childless adults aged 19 to 64 with income below $100 \%$ of the federal poverty level. $N=80,020$; exact sample size differs for each outcome due to missing data for certain individuals or certain years. Column (1) displays regression-adjusted difference-in-differences estimate for impact of Medicaid expansion on outcome. Regression models adjusted for individual demographic characteristics, state unemployment rate, state of residence, and year of interview. Estimates account for BRFSS survey weights, and standard errors are clustered by state. 95\% confidence intervals are in brackets. Column (2) displays the P value for the DD estimate. Column (3) displays pre-2014 means for outcome variable in Medicaid expansion states, adjusted by BRFSS survey weights

implementation, Medicaid expansions increased the probability of having insurance by 10.3 percentage points $(95 \% \mathrm{CI}$ $0.067,0.140 ; P<0.001)$ or $16.7 \%$. The expansions also improved access to care: the probability of having a personal doctor increased by 2.5 percentage points $(95 \% \mathrm{CI} 0.001$, $0.049 ; P=0.044$ ) or $3.9 \%$, and the probability that the cost was a barrier to medical care declined by 3.6 percentage points $(95 \% \mathrm{CI}-0.058,-0.014 ; P=0.002)$ or $10.7 \%$.

Medicaid expansions also increased the probability of receiving certain preventive care. The probability of ever having an HIV test rose by 2.5 percentage points $(95 \% \mathrm{CI} 0.0003$, $0.050 ; P-0.048)$ or $5.9 \%$, and the probability of having had a dentist visit in the past 12 months rose by 2.5 percentage points $(95 \% \mathrm{CI} 0.0001,0.050 ; P=0.050)$ or $5.5 \%$. There was no detectable impact on routine checkups, flu shots, breast exams, pap tests, or mammograms.

There was also no detectable effect of expansion on risky health behaviors such as smoking, alcohol abuse, exercise, BMI, or obesity.

Medicaid expansions were associated with improvements in several measures of self-assessed health, including an increase in general health (on a 5-point scale) of 0.089 points (95\% CI $0.050,0.129 ; P<0.001)$ or $3.0 \%$, a reduction days in the past month spent in poor mental health of 0.704 days (95\%CI $-1.196,-0.211 ; P=0.006)$ or $8.6 \%$, and a decline in days in the past month during which poor health prevented usual activities of 0.969 days $(95 \% \mathrm{CI}-1.563,-0.375 ; P=$ 0.002 ) or $10.1 \%$.

\section{DISCUSSION}

The benefits of Medicaid expansions on insurance coverage, access to care, HIV tests, dental visits, and self-assessed health persisted beyond the first 2 years after expansion. ${ }^{1-6}$ Comparing this paper's estimates of the 3-year impacts with our earlier estimates of the 2-year impacts ${ }^{2}$, we find that they are not significantly different. The point estimates of the impacts for the first 3 years tend to be slightly smaller than those for the first 2 years (compare the results in Table 1, column 8 of Simon, Soni, and Cawley, 2017, to those in Table 1 of this paper). ${ }^{2}$ Although this difference is not statistically significant, it does indicate the importance of continuing to monitor and evaluate the effects of the expansions to see if the benefits may be changing over time.

The persistence of benefit from the Medicaid expansions is noteworthy and important. Past studies of the shorter-term impacts of the program acknowledged that changes in health and utilization may take longer than a year or two to materialize and that it may have been too soon for them to observe the full effects of the Medicaid expansions, or called for continued monitoring to ensure that the benefits that they detected persisted in later years. ${ }^{1}{ }^{1}$ This study confirms that the increases in insurance coverage, access, preventive care, and overall health that were found earlier have indeed persisted into the third year of the program.

In many cases, the estimated effects were large. Medicaid expansion increased the probability of having a personal 
doctor by $3.9 \%$, decreased the probability that cost was a barrier to medical care by $10.7 \%$, increased the probability of having an HIV test by $5.9 \%$, increased the probability of a dental visit by $5.5 \%$, improved self-assessed health by $3.0 \%$, reduced the number of days with poor mental health by $8.6 \%$, and reduced the number of days in which poor health prevented usual activities by $10.1 \%$. Given that the expansions increased health insurance coverage by 10.3 percentage points, the effects for those who actually gained health insurance as a result of the expansion should be about nine times larger than the estimates listed in Table 1. In some cases, the effects may seem implausibly large. However, while the expansions increase the probability that an individual has health insurance at the time of the interview by 10.3 percentage points, it is likely that additional low-income childless adults benefitted from the Medicaid expansion at some other point in the past 3 years, and are healthier as a result. That is, there may be churning in which individuals are covered by the program, and thus a larger percentage of the eligible population than the 10.3 percentage point increase at a single point in time likely benefitted from the program, which may partly explain the large estimated benefits.

Consistent with our earlier study based on 2 years of postexpansion data ${ }^{2}$, this study based on 3 years of post-expansion data finds no detectable impact of the expansions on risky health behaviors such as smoking, alcohol abuse, or obesity.

Limitations of the study include the BRFSS is a repeated cross section so it is not possible to observe changes in the same individuals, it does not record the source of insurance, and it contains only certain measures of preventive care and access to care. Future research should continue to monitor outcomes in expansion states relative to non-expansion states to track the longer-term effects of Medicaid expansion.

Acknowledgements: Cawley received financial support from the Robert Wood Johnson Foundation (RWJF) through an Investigator Award in Health Policy Research but the RWJF played no role in the design and conduct of the study. All authors had full access to the data in the study and take responsibility for the integrity of the data and the accuracy of the data analysis.

Corresponding Author: John Cawley, Ph.D.; Department of Policy Analysis and Management Cornell University, Ithaca, NY, USA (e-mail: JHC38@cornell.edu).

\section{Compliance with Ethical Standards:}

Conflict of Interest: The authors declare that they do not have a conflict of interest.

\section{REFERENCES}

1. Miller S, Wherry LR. Health and Access to Care during the First 2 Years of the ACA Medicaid Expansions. N Engl J Med 2017;376(10):947-56.

2. Simon K, Soni A, Cawley J. The Impact of Health Insurance on Preventive Care and Health Behaviors: Evidence from the First Two Years of the ACA Medicaid Expansions. J Policy Anal Manag 2017;36(2):390-417.

3. Wehby GL, Lyu W. The Impact of the ACA Medicaid Expansions on Health Insurance Coverage through 2015 and Coverage Disparities by Age, Race/Ethnicity, and Gender. Health Serv Res 2017;Early View.

4. Decker SL, Lipton BJ, Sommers BD. Medicaid Expansion Coverage Effects Grew In 2015 With Continued Improvements In Coverage Quality. Health Aff 2017;36(5):819-25.

5. Antonisse L, Garfield R, Rudowitz R, Artiga S. The Effects of Medicaid Expansion under the ACA: Updated Findings from a Literature Review. 2017.

6. Courtemanche C, Marton J, Ukert B, Yelowitz A, Zapata D. Early Effects of the Affordable Care Act on Health Care Access, Risky Health Behaviors, and Self-Assessed Health. South Econ J [Internet] 2018;84(3):660-91. Available from: http://doi.wiley.com/10.1002/soej. 12245 